\title{
Visual Circuit Assembly Requires Fine Tuning of the Novel Ig Transmembrane Protein Borderless
}

\author{
Scott Cameron, ${ }^{1}$ Wen-Tzu Chang, ${ }^{1}$ Yixu Chen, ${ }^{1}$ Yating Zhou, ${ }^{3}$ Shaurya Taran, ${ }^{3}$ and Yong Rao ${ }^{1,2}$ \\ ${ }^{1}$ Department of Neurology and Neurosurgery, McGill Centre for Research in Neuroscience and Departments of ${ }^{2}$ Medicine and ${ }^{3}$ Biology, McGill University \\ Health Centre, Montreal, Quebec H3G 1A4, Canada
}

Establishment of synaptic connections in the neuropils of the developing nervous system requires the coordination of specific neuriteneurite interactions (i.e., axon-axon, dendrite-dendrite and axon-dendrite interactions). The molecular mechanisms underlying coordination of neurite-neurite interactions for circuit assembly are incompletely understood. In this report, we identify a novel Ig superfamily transmembrane protein that we named Borderless (Bdl), as a novel regulator of neurite-neurite interactions in Drosophila. Bdl induces homotypic cell-cell adhesion in vitro and mediates neurite-neurite interactions in the developing visual system. Bdl interacts physically and genetically with the Ig transmembrane protein Turtle, a key regulator of axonal tiling. Our results also show that the receptor tyrosine phosphatase leukocyte common antigen-related protein (LAR) negatively regulates Bdl to control synaptic-layer selection. We propose that precise regulation of Bdl action coordinates neurite-neurite interactions for circuit formation in Drosophila.

\section{Introduction}

The presence of numerous axons and dendrites in the neuropils of the developing CNS makes it a daunting task for establishing specific synaptic connections. Studies over the last two decades have identified a number of cell-surface recognition molecules that mediate specific neurite-neurite interactions for circuit assembly (Giagtzoglou et al., 2009). That many cell-surface recognition molecules are present broadly in developing neuropils throughout embryonic development, however, raises the question how the action of cell-surface recognition molecules is modulated temporally to ensure accuracy in circuit formation.

The assembly of visual circuits in Drosophila is an attractive model for understanding the general mechanisms underlying spatiotemporal control of neurite-neurite interactions (Hadjieconomou et al., 2011; Melnattur and Lee, 2011). The Drosophila adult visual system is comprised of the compound eye and the optic lobe (Meinertzhagen and Hanson, 1993). The compound eye consists of $\sim 800$ ommatidia, each containing six outer photoreceptor neurons (R1-R6) for processing motion and two inner photoreceptor neurons (R7 and R8) for processing color. R1-R6 axons form synaptic connections in the superficial lamina layer, and R7 and R8 axons project through the lamina into the deeper medulla layer, where they are organized into $\sim 800$ regu-

\footnotetext{
Received May 4, 2013; revised Aug. 29, 2013; accepted Sept. 26, 2013.

Author contributions: S.C., W.-T.C., Y.C., Y.Z., S.T., and Y.R. designed research; S.C., W.-T.C., Y.C., Y.Z., and S.T. performed research; S.C., W.-T.C., Y.C., Y.Z., S.T., and Y.R. analyzed data; S.C. and Y.R. wrote the paper.

This work was supported by Canadian Institutes of Health Research Operating Grant MOP-14688 (Y.R.) and a McGill University Health Centre studentship (S.C.). We thank Dr. S. Carbonetto for critically reading this manuscript, and the Bloomington Stock Center, the Drosophila Genomic Resource Center, Dr. C. Lee, Dr. F. Shoeck, Dr. I. Salecker, and Dr. J. Treisman for reagents and fly stocks.

The authors declare no competing financial interests.

Correspondence should be addressed to Dr. Yong Rao, Centre for Research in Neuroscience, McGill University Health Centre, Room L7-136, 1650 Cedar Avenue, Montreal, Quebec H3G 1A4, Canada. E-mail:yong.rao@mcgill.ca. DOI:10.1523/JNEUROSCI.1878-13.2013

Copyright $\odot 2013$ the authors $\quad 0270-6474 / 13 / 3317413-09 \$ 15.00 / 0$
}

larly spaced columns. Each R7 and R8 axon from the same ommatidium terminate in a topographic manner in two synaptic layers within the same column. The R8 axon terminates within the M3 layer, and the R7 axon terminates in the deeper M6 layer.

Visual circuit assembly in Drosophila involves complex neurite-neurite interactions. Specific recognition between R-cell axons and their target layers in the optic lobe have been shown to be required for synaptic-layer selection (Lee et al., 2001, 2003; Maurel-Zaffran et al., 2001; Prakash et al., 2005; Shinza-Kameda et al., 2006; Timofeev et al., 2012). Visual circuit assembly also requires the interactions among $\mathrm{R}$-cell axons. Selection of postsynaptic targets by R1-R6 axons in the lamina requires specific axon-axon interactions (Clandinin and Zipursky, 2000). The assembly of medulla columns requires modulation of both heterotypic and homotypic axon-axon adhesion (Sanes and Zipursky, 2010). For instance, receptor tyrosine phosphatases LAR and protein tyrosine phosphatase 69D (PTP69D) are reported to be involved in negatively regulating the adhesion between R7 and R8 axons for facilitating R7 synaptic-layer selection (Newsome et al., 2000; Maurel-Zaffran et al., 2001). And Ig-superfamily transmembrane proteins Dscam2 and Turtle (Tutl) prevent homotypic axon-axon terminal adhesion for tiling L1 (Millard et al., 2007) and R7 axons (Ferguson et al., 2009), respectively. The exact mechanisms by which those cell-surface recognition molecules negatively regulate axon-axon adhesion, however, remain unknown.

In the present study, we investigate the role of a novel Ig-superfamily transmembrane protein Borderless (Bdl) in Drosophila. Bdl is expressed in the developing visual system, and functions as a cell-surface recognition molecule to mediate neurite-neurite interactions. The receptor tyrosine phosphatase LAR and the Ig-superfamily transmembrane protein Tutl are key regulators of Bdl-mediated axon-axon interactions in controlling synaptic-layer selection and axonal tiling, respectively. Our results 
shed new light on spatiotemporal control of cell-surface recognition molecules for coordinating circuit assembly.

\section{Materials and Methods}

Genetics. KG06404 was obtained from the Bloomington Stock Center. $b d l^{\mathrm{EX} 1}$ and $b d l^{\mathrm{EX} 2}$ deletion alleles were generated by imprecise excision of KG06404. In the $b d l^{\text {EX1 }}$ allele, the sequence encoding amino acids 539-719 was deleted. In the $b d^{\mathrm{EX} 2}$ allele, the sequence encoding amino acids $317-719$ was deleted. $p B a c[\mathrm{WH}]$ [f03313] and pBac[WH]CG16857[f02225] were used to generate $b d l$ and tutl double mutants by using the FLP/FRT-based strategy described previously (Parks et al., 2004). The entire tutl gene and most of the $b d l$ gene encoding the amino acid sequence 1-542 of Bdl polypeptide were deleted in double mutants. Deletion was confirmed by complementation test, PCR, and DNA sequencing analysis.

Genetic experiments were performed with animals of either sex. Large clones ( $>90 \%$ of retina) of homozygous tutl ${ }^{01085}$ single-mutant or tutl/ $b d l$ double-mutant tissues were generated in an otherwise heterozygous or wild-type eye by eyespecific mitotic recombination using the eyFLP/ FRT system (Newsome et al., 2000). To reduce the dosage of $b d l$ by $50 \%$ in tutl mutants, genetic crosses were performed to generate flies with the genotype $t u t l, b d l$ (i.e., double mutant)/tutl $l^{23},+$. To specifically label R7 axons in flies overexpressing $b d l$, genetic crosses were performed to generate Rh4-GFP/GMR-GAL4; UAS-bdl-Flag progeny. To examine genetic interactions between $L A R$ and $b d l$, flies with the genotype $L A R^{\mathrm{c} 12}, b d l^{\mathrm{EX} 2} / L A R^{2127}$ were generated. To overexpress LAR in flies with mild Bdl overexpression, flies with the genotype GMR-GAL4, UAS-bdl-Flag (mild)/UAS$H A-L A R$ were generated. To examine potential genetic interactions between $P T P 69 D$ and $b d l$, flies with the genotype eyFLP; $b d l^{\mathrm{EX} 2} /+; P T P 69 D^{\mathrm{d} 1689}$, FRT80B/M(3)RpS174 $P[w+] \quad F R T 80 B$ or eyFLP; $b d l^{\mathrm{EX} 2} / b d l^{\mathrm{EX} 2}$; PTP69D ${ }^{\mathrm{d} 1689}$, FRT80B/M(3)RpS174 P[w+] FRT80B were generated.

Molecular biology. The complete $b d l$ coding sequence was amplified by PCR using the GH11322 EST clone as the template. The resulting PCR product was directionally subcloned into pENTR/D-TOPO (Invitrogen) and subsequently subcloned into the pUAST expression vector using LR clonase (for addition of either C-terminal Venus, monomeric RFP, or FLAG tags) (provided by Drosophila Genomics Resource Center). To generate LAR- $\Delta$ cyto construct, DNA sequence encoding amino-acid sequence from $\mathrm{N}$-terminal to 1412 was amplified by PCR using UAS-HA$L A R$ as the template, and then subcloned into the pUAST expression vector similarly as above using the Drosophila Gateway system (Invitrogen). Bdl deletion constructs were generated from the pENTR-Bdl construct. An AvrII restriction site was placed into PCR primers for generating different domain deletions, which were then subcloned into the pUAST expression vector by using the Drosophila Gateway system. Resulting domain-deletion constructs include $\Delta \operatorname{Ig} 1$ (Asn27 to Ser128), $\Delta$ Ig2 (Ser128 to Ile233), $\Delta \operatorname{Ig} 1-2$ (Asn42 to Ile233), $\Delta \operatorname{Ig} 3-4$ (Tyr259 to Ala415), and $\Delta$ FN1-2 (Ala434 to Ser636). For domain swapping, the DNA fragment encoding the two Ig domains of PTP69D (Ser29 to Thr230) was subcloned into the $b d l$ expression construct to replace the region encoding Ig1 and Ig2 domains (Asn42 to Ile233) of Bdl. Expression of Bdl domain-deletion proteins in transfected cells was confirmed by both immunostaining and Western blot analysis. To create a Bdl-Fc fusion construct, the sequence encoding for the entire extracellular region of Bdl was amplified by PCR and then subcloned into the PIB/Fc expression vector for pro-
PM
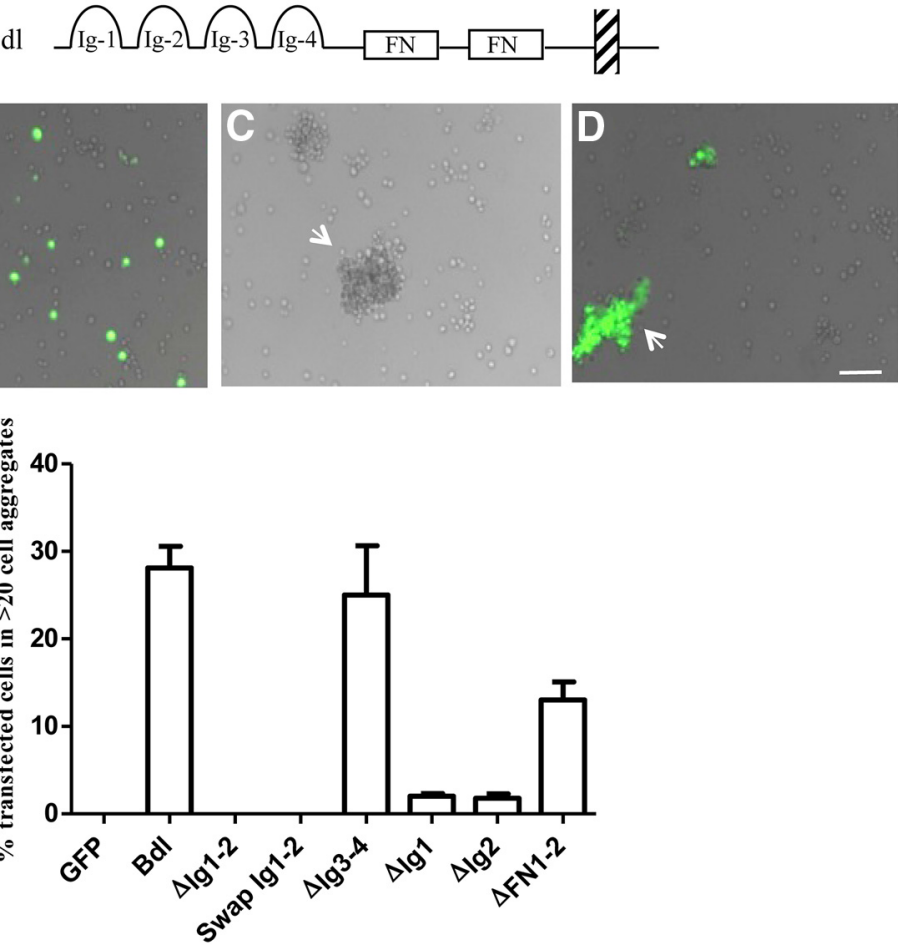

Figure 1. Bdl mediates homotypic cell-cell adhesion. $\boldsymbol{A}$, Domain structure of Bdl. FN, Fibronectin type-III repeat; PM, plasma Ig 1-2 construct, Ig1 and lg2 domains (Asn42 to lle233) of Bdl were replaced with the two lg domains of PTP69D (Ser29 to Thr230). Three independent experiments were performed. Scale bar, $40 \mu \mathrm{m}$. Error bars indicate SEM.

ducing the Bdl-Fc fusion protein in S2 cells. The Bdl-Fc fusion protein was purified using a Protein A-conjugated Sepharose column, and used to raise anti-Bdl antibody in rabbits.

Histology. Cryostat sections of pupal and adult heads were stained as described previously (Ferguson et al., 2009). Antibodies were used at the following dilutions: MAb24B10, 1:100 (Developmental Studies Hybridoma Bank); rabbit polyclonal anti-GFP, 1:750 (Invitrogen); rabbit polyclonal anti-Bdl, 1:1000. Secondary antibodies (Invitrogen) were used at a 1:500 dilution.

Cell aggregation. Drosophila S2 cells were grown in EX-Cell 420 SerumFree Medium for Insect Cells (Sigma) at $25^{\circ} \mathrm{C}$. Cells $\left(1 \times 10^{7} / 10 \mathrm{ml}\right)$ were transfected with $2 \mu \mathrm{g}$ of total plasmid DNA $(0.2 \mu \mathrm{g}$ actin-GAL4 and 1.8 $\mu \mathrm{g} U A S$ construct) with Effectene (Qiagen). At $96 \mathrm{~h}$ after transfection, cells were prepared as single-cell suspension at a concentration of $2.25 \times$ $10^{6} / \mathrm{ml}$ and agitated at $150 \mathrm{rpm}$ for $1 \mathrm{~h}$ (aggregation) or $3 \mathrm{~h}$ (coaggregation) at room temperature. One hundred and fifty microliters of cell mixture were then transferred onto glass slides (Fisher Scientific). Total GFP- or RFP-positive transfected cells in 10 randomly selected fields were counted. The number of GFP- or RFP-positive transfected cells forming large cell aggregates ( $>20$ cells) in each field were also counted to determine the percentage of transfected cells that formed large cell aggregates. In coaggregation experiments, a cell coaggregate is defined as a cell aggregate $(>20$ cells) in which the percentage of each cell population (i.e., GFP- or RFP-positive cells) should be at least 15\%.

Statistical analysis. Two-tailed $t$ tests were used for statistical analysis. The difference is considered significant when the $p$ value is $<0.05$.

\section{Results}

Bdl mediates homotypic cell-cell adhesion in vitro

In a search for cell-surface recognition molecules regulating circuit development, we characterized the novel transmembrane protein 

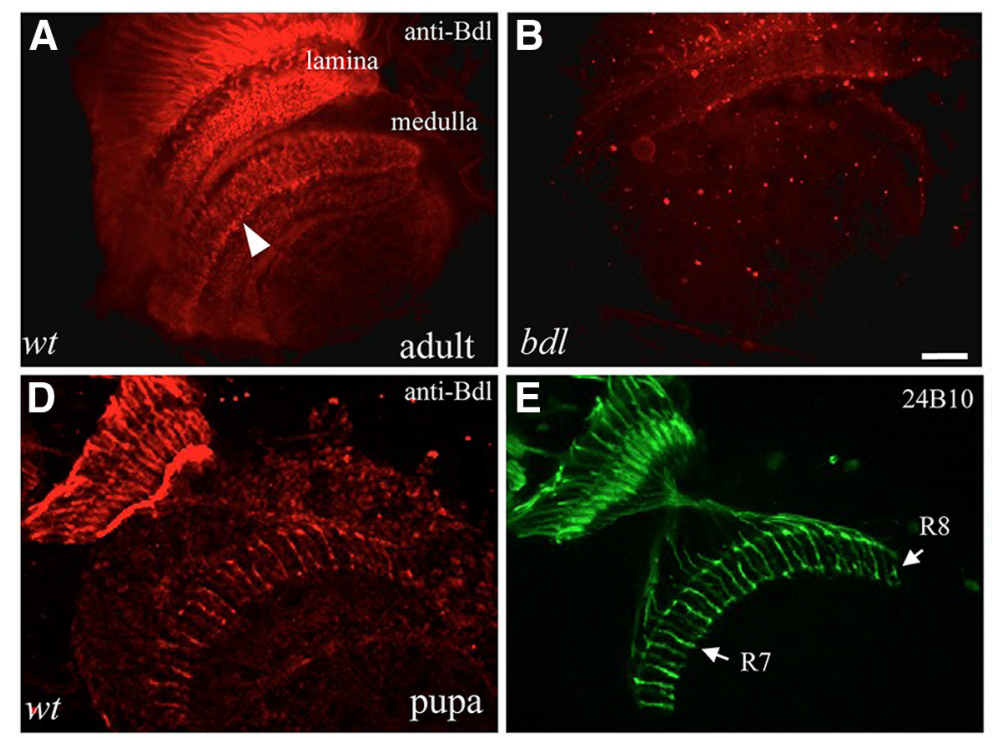

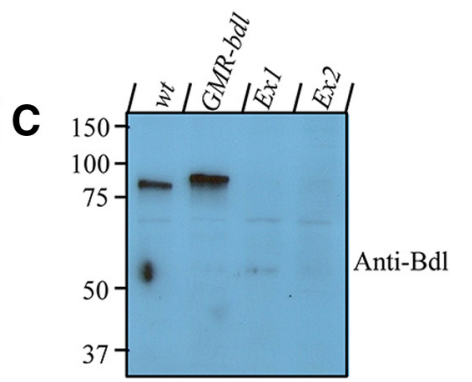

$-\infty=$ Anti-Actin

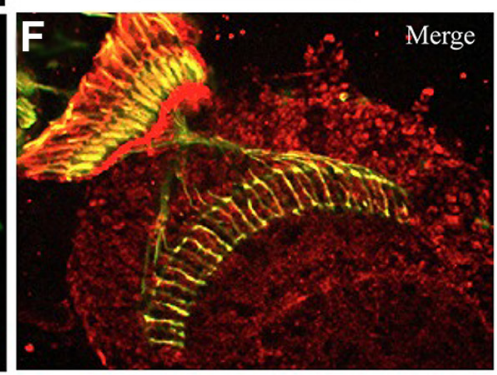

Figure 2. Bdl is expressed in the Drosophila visual system. $\boldsymbol{A}, \boldsymbol{B}$, Frozen sections of adult heads were stained with anti-Bdl antibody. $\boldsymbol{A}$, In wild type, Bdl was detected in lamina and medulla. Within the medulla, strong staining at the R7 terminal layer was detected (arrowhead). $\boldsymbol{B}$, Bdl staining was absent in $b d l$ deletion mutants (i.e., $b d l{ }^{\mathrm{EX} 2}$ ). $\boldsymbol{C}$, Consistent with the predicted size of Bdl, anti-Bdl detected an $\sim 80 \mathrm{kDa}$ band in wild-type and Bdl-overexpression (i.e., GMR-bdl) head lysates, which was absent in lyastes made from $b d l{ }^{\mathrm{EX} 1}$ and $b d l^{\mathrm{EX} 2}$ mutant flies. The same blot was probed with anti-Actin antibody as a loading control. $\mathbf{D}-\boldsymbol{F}$, Frozen sections of wild-type fly heads at the $50 \%$ pupal stage were double-stained with anti-Bdl (red) and MAb 24B10 (green). The arrows in $\boldsymbol{E}$ indicate R7 and R8 terminals. Scale bar, $20 \mu \mathrm{m}$.
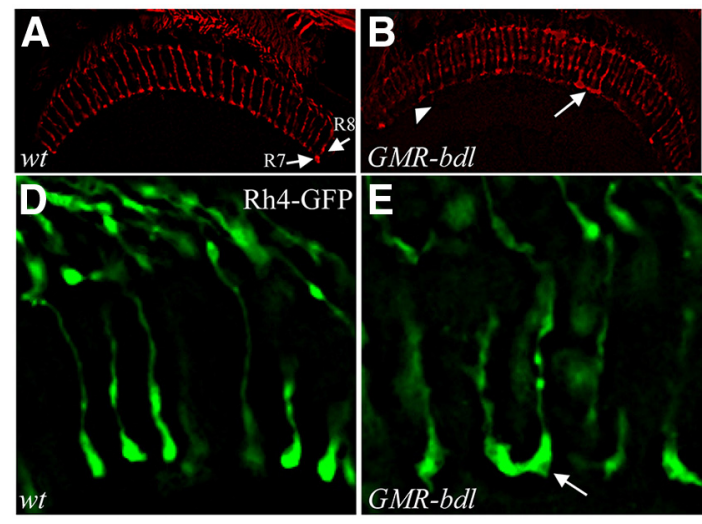

G

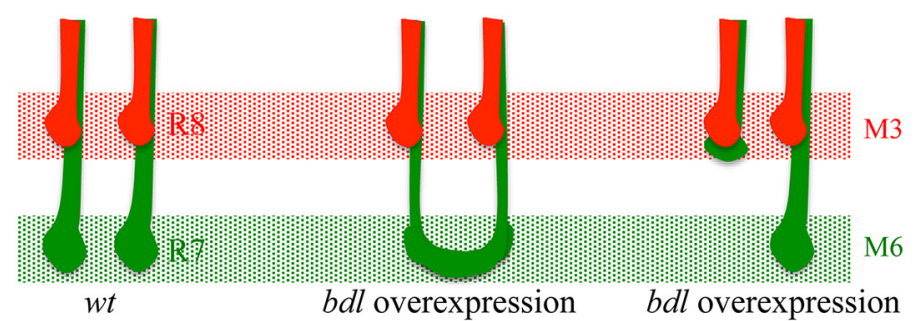

Figure 3. Overexpression of Bdl disrupted R7 axonal tiling and synaptic-layer selection. $\boldsymbol{A}-\boldsymbol{C}$, The projection pattern of all R-cell axons in frozen sections of adult heads were visualized either with R-cell-specific MAb 24B10 $(\boldsymbol{A}, \boldsymbol{B})$ or with anti-FLAG that stains all R-cells axons in flies carrying GMR-GAL4 and UAS-bdl-Flag (C). $\boldsymbol{A}$, In wild type, the frequencies of R7 tiling (i.e., abnormal R7 terminal adhesion) and R7 targeting errors in wild-type flies are only $\sim 2-4 \%$ of R7 axons and $<1 \%$ of R7 axons, respectively. $n$ $>400$. Arrows indicate R7 and R8 terminals. $B$, In flies with mild $b$ dl overexpression in R-cell axons, abnormal adhesion (arrow) between neighboring $R 7$ terminals and mistargeting of $R 7$ axons into the $R 8$ terminal layer (arrowhead) were observed. The level of low versus high Bdl expression was based on Bdl immunostaining (data not shown). C, Flies with strong Bdl overexpression. Only one R7 axon (arrow) terminated correctly in this section. $\mathbf{D}-\boldsymbol{F}, \mathrm{R} 7$ axons in adult heads were labeled by the R7-specific marker Rh4-GFP. D, Wild type. $\boldsymbol{E}$, In a fly overexpressing $b d l$, two adjacent R7 terminals stick with each other (arrow). $\boldsymbol{F}$, In a fly overexpressing $b d l$, many R7 axons mistargeted to the R8 terminal layer (arrowheads). G, Schematic summary of the Bdl overexpression phenotype. Scale bars: (in $\boldsymbol{C}) \boldsymbol{A}-\boldsymbol{C}, 20 \mu \mathrm{m}$; (in F) $\boldsymbol{D}-\boldsymbol{F}, 5 \mu \mathrm{m}$. encoded by CG16857. CG16857 consists of an extracellular region containing four Iglike domains and two fibronectin-type-III repeats, a single transmembrane region, and a cytoplasmic domain (Fig. 1A). The extracellular region of CG16857 displays significant homology to Ig-superfamily members such as Tutl/Dasm1/IgSF9 ( $\sim 31.6 \%$ identity), Frazzled/DCC/UNC-40 ( $\sim 23.7 \%$ identity), Robo ( $\sim 25.9 \%$ identity), and Dscam2 ( $24.6 \%$ identity). We name the CG16857 gene borderless ( $b d l)$, based on the phenotypes caused by Bdl misregulation.

To test whether Bdl plays a role in mediating cell-surface recognition, we performed cell culture studies. Bdl was expressed in the Drosophila Schneider-2 cells (S2 cell). Untransfected cells or cells transfected with the GFP expression construct did not induce the formation of large cell aggregates ( $>20$ cells; Fig. 1B). Bdl, like N-cadherin (Fig. 1C), induced the formation of large cell aggregates ( $>20$ cells; Fig. 1D,E). Each large cell aggregate consisted predominantly of cells expressing Bdl ( $>85 \%$ of cells in each aggregate), indicating that Bdl-expressing cells selectively aggregated with each other, but not with untransfected cells (Fig. 1D). This result suggests that Bdl possesses homophilic binding activity. To determine domain requirements, we performed deletion analysis. Deletion of Ig1 and Ig2 domains of Bdl or swapping Ig1 and Ig2 of Bdl with the 
Ig domains of PTP69D completely abolished adhesive activity (Fig. $1 E$ ) without affecting the expression level or cell-surface presence of mutant Bdl proteins (data not shown). These results indicate that both Ig1 and Ig2 domains are required for mediating cell-cell aggregation.

\section{Bdl is expressed in the developing Drosophila visual system}

To determine the role of $\mathrm{Bdl}$, we examined the expression pattern of $\mathrm{Bdl}$ in the Drosophila visual system. We performed immunostaining using an anti-Bdl antibody raised against a Bdl-Fc fusion protein, which contains the entire extracellular region (see Materials and Methods). In wildtype adult flies, Bdl staining was detected in lamina, medulla, and inner optic lobe (Fig. $2 A)$. The specificity of this antibody was supported by the absence of staining in $b d l$ mutants (Fig. 2B) and also by Western blot analysis $(C)$. Strong Bdl staining was also detected in the developing lamina, medulla, and inner optic lobe at 50\% pupal stage (Fig. $2 D, F)$. Within the medulla, Bdl was detected in R7 and R8 axonal terminals, and other neuronal processes (Fig. 2D,F). Thus, $\mathrm{Bdl}$ is present in the developing optic lobe during the establishment of R-cell connection pattern.
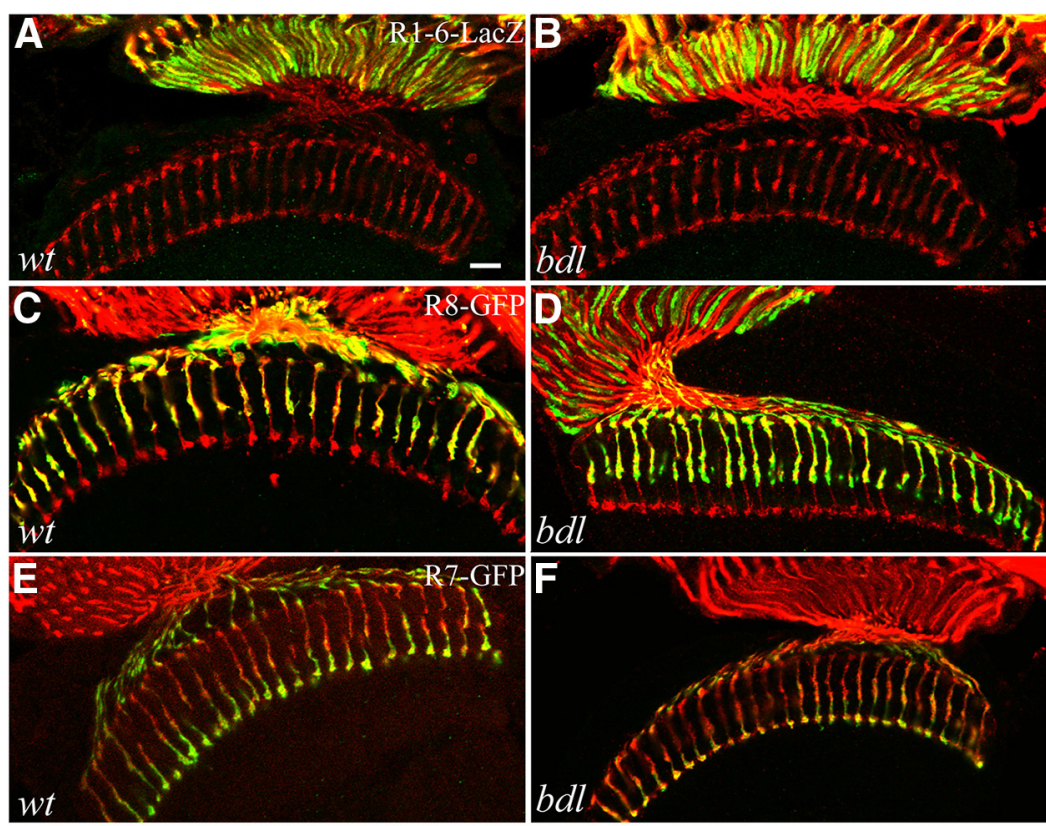

Figure 4. Loss of $b d l$ did not affect R-cell synaptic-layer selection and tiling. The R-cell axonal projection pattern in frozen sections of adult fly heads was visualized with $24 B 10$ (red) for labeling all R-cell axons and subtype-specific R-cell markers (green). $\boldsymbol{A}, \boldsymbol{B}$, The R1-R6 projection pattern was visualized with R1-R6-specific expression of UAS-LacZ driven by Rh1-GAL4. $\boldsymbol{A}$, In wild type,

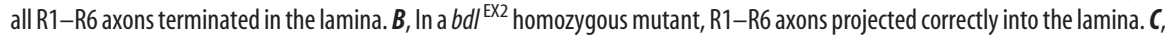
D, R8 projection pattern was visualized with R8-specific expression of UAS-GFP driven by Rh5, 6-GAL4. C, In wild type, R8 axons select the M3 layer for synaptic connection. D, No obvious defect in R8 synaptic-layer selection was observed in bdl ${ }^{\mathrm{EX} 2}$ homozygous mutants. $\boldsymbol{E}, \boldsymbol{F}, \mathrm{R} 7$ projection pattern was visualized with R7-specific expression of UAS-GFP driven by PanR7-GAL4. $\boldsymbol{E}$, In wild type, R7 axons select the M6 layer for synaptic connection. $\boldsymbol{F}$, R7 axons correctly terminated at the M6 layer in bdl ${ }^{\text {EX2 }}$ homozygous mutants. The results are summarized in Table 1 . Scale bar, $10 \mu \mathrm{m}$.

\section{Overexpression of Bdl disrupted R7 synaptic-layer selection and axonal tiling}

To determine the potential role of $\mathrm{Bdl}$ in mediating neurite-neurite interactions, Bdl was overexpressed in R-cell axons under control of the eye-specific driver GMR-GAL4. In wild type, R7 and R8 axons form a regularly spaced columnar projection pattern and terminate in two separate synaptic layers, M6 and M3, respectively (Fig. 3A). In flies overexpressing $b d l$, R7 axonal terminals frequently stick with R7 terminals in neighboring columns (Fig. $3 B, E, G$ ), and many R7 axons terminated abnormally at the R8 terminal layer (Fig. $3 B, C, F, G$ ). The severity of the phenotypes varied among UAS-bdl transgenic lines. Transgenic flies with mild Bdl overexpression showed both R7 tiling (i.e., abnormal R7-R7 terminal adhesion) and R7 mistargeting phenotypes ( $~ 10-30 \%$ of R7 axons; $n>400$; Fig. $3 B, E, F)$. Whereas transgenic flies with strong $\mathrm{Bdl}$ overexpression displayed only the R7 mistargeting phenotype with high penetrance ( $\sim 80-95 \%$ of R7 axons; $n>400$; Fig. $3 C$ ). The R7 tiling phenotype caused by Bdl overexpression (Fig. $3 B, E$ ), together with Bdl-induced homotypic adhesion in cultured cells (Fig. $1 D, E$ ), indicates that Bdl increased the adhesion between R7 axonal terminals in adjacent columns. Similarly, overexpression of Bdl also increased adhesion between R7 and R8 axons within the same column. An increase in R7-to-R8 adhesion could interfere with the separation of R7 axon from the R8 axon, a process necessary for proper targeting of R7 axons (Newsome et al., 2000; Maurel-Zaffran et al., 2001). We conclude that Bdl is capable of promoting neurite-neurite interactions.

Loss of $b d l$ did not affect R-cell axonal tiling and synapticlayer selection

To determine the in vivo function of Bdl, we performed loss-offunction analysis. $b d l$ deletion alleles were generated by imprecise
Table 1. R-cell axonal tiling and synaptic-layer selection remained normal in bdl mutants

\begin{tabular}{llllll}
\hline Genotype & Markers & $\begin{array}{l}\text { Animals } \\
\text { examined }\end{array}$ & $\begin{array}{l}\text { Columns } \\
\text { examined }\end{array}$ & $\begin{array}{l}\text { Mistargeted } \\
\text { axons }\end{array}$ & $\begin{array}{l}\text { Abnormal terminal } \\
\text { adhesion }\end{array}$ \\
\hline$w t$ & R1-R6 & 5 & 280 & 0 & ND \\
$b d l^{E X 2}$ & $R 1-R 6$ & 5 & 285 & 0 & ND \\
$w t$ & $R 7$ & 5 & 233 & 2 & 4 \\
$b d l^{E X 2}$ & $R 7$ & 6 & 341 & 0 & 11 \\
$w t$ & R8 & 8 & 311 & 10 & 0 \\
$b d l^{E X 2}$ & R8 & 7 & 386 & 9 & 3 \\
\hline
\end{tabular}

$w t$, Wild type; ND, Not determined.

excision of a P-element sequence inserted into the region immediately downstream of the $b d l$ gene (see Materials and Methods). Two deletion alleles appear to be protein null alleles, as no Bdl protein could be detected in mutants by Western blot or immunostaining analysis (Fig. $2 B, C$ ). We performed phenotypic analysis to examine the effects of $b d l$ mutations on R-cell connection pattern. Cell-type-specific markers were used to examine the projection pattern of R1-R6, R7, and R8. Neither synaptic-layer selection of R1-R6, R7, and R8 axons, nor tiling of R7 and R8 axons, was affected in $b d l$ mutants (Fig. 4 ; Table 1 ). We conclude that $\mathrm{Bdl}$ is not absolutely required for R7 and R8 axonal tiling and synaptic-layer selection.

\section{Tutl interacts with $\mathrm{Bdl}$ in controlling $\mathrm{R} 7$ axonal tiling}

That overexpression of Bdl caused an R7 axonal tiling phenotype (Fig. 3) raises the interesting possibility that R7 tiling involves downregulation of Bdl-mediated adhesion between adjacent R7 axonal terminals. In our previous studies, we showed that Tutl is required for R7 axonal tiling, but not required for synaptic-layer 
A

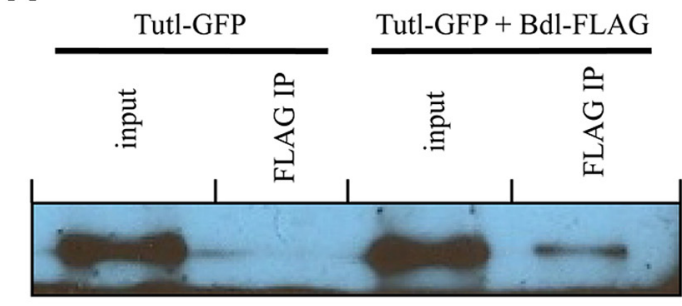

\section{LAR-HA + Bdl-FLAG}
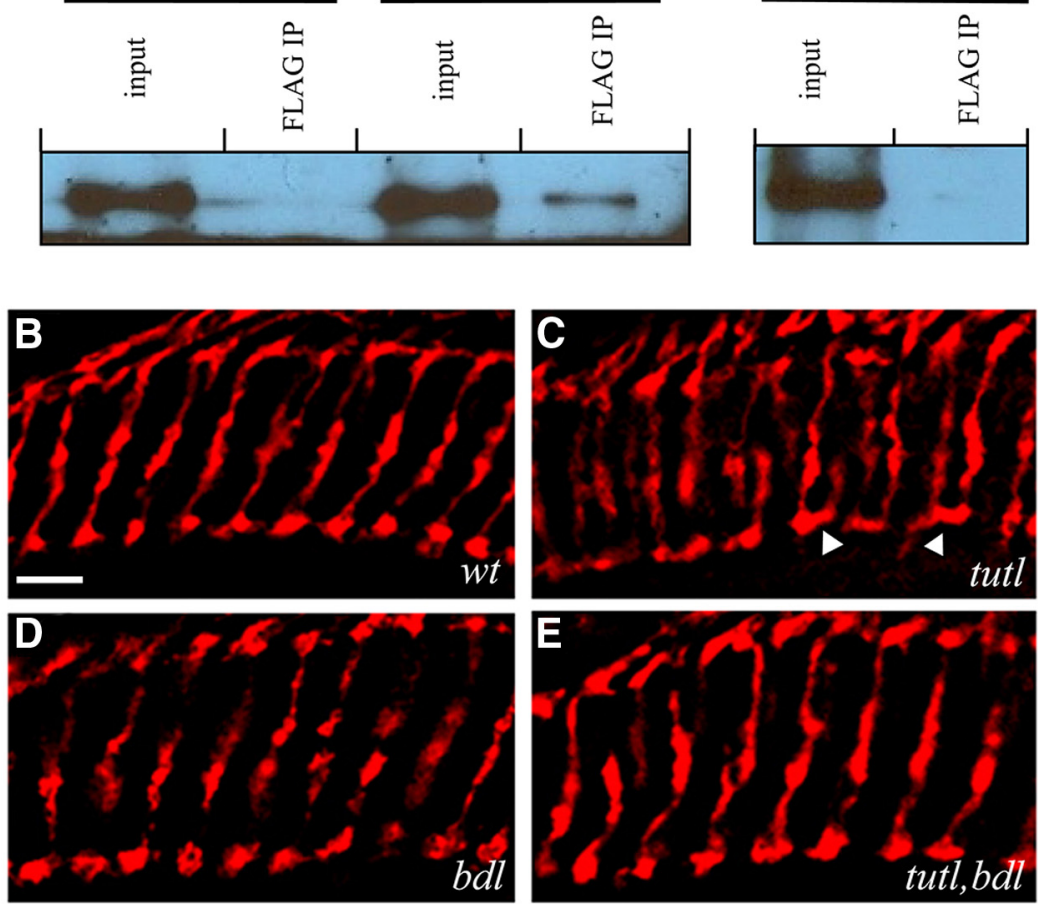

$\mathbf{F}$

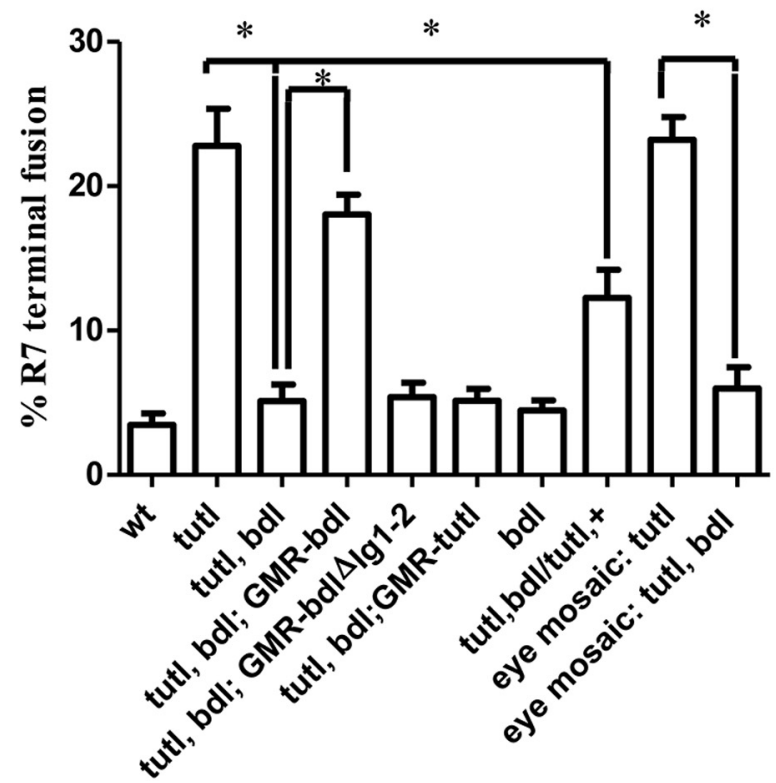

Figure 5. Tutl interacts physically and genetically with Bdl. $A$, Tutl associates with Bdl in cultured cells. Anti-GFP antibody detected Tutl-GFP in anti-FLAG precipitates from cell lysates coexpressing Tutl-GFP and Bdl-FLAG. No Tutl-GFP was detected in anti-FLAG precipitates from cell lysates expressing Tutl-GFP only. No LAR-HA was detected by anti-HA antibody in anti-FLAG precipitates from cell lysates coexpressing LAR-HA and Bdl-FLAG. $\boldsymbol{B}-\boldsymbol{E}$, R-cell axonal projections in adult heads were visualized with MAb 24B10 staining. $\boldsymbol{B}$, Wild type. $\boldsymbol{C}$, In homozygous tut ${ }^{23}$ mutants, many R7 terminals stick with neighboring R7 terminals (arrowheads). D, R7 tiling pattern was normal in bdl null mutants (i.e., bdl ${ }^{\mathrm{EX} 2}$ ). E, R7 tiling pattern in homozygous tutl and $b d l$ double mutants was normal. $\boldsymbol{F}$, Quantitation of the R7 tiling phenotype in flies with different genotypes. The number of animals examined in each genotype is as follows: wild type (wt), 11; tut ${ }^{23}, 5$; tutl, bdl, 5; tutl, bdl; longGMR-bdl, 6; tutl, bdl; longGMRbdl $\Delta / g 1-2,6 ;$ tutl, bdl; longGMR-tutl, 6; bdl ${ }^{\mathrm{Ex} 2}, 11 ;$ tutl, bdl/tut $\left.\right|^{23}, 6 ;$ tutl $^{1085}$ eye mosaic, 5; tutl, bdl eye mosaic, $4 .{ }^{*} p<0.01$. Scale bar: $5 \mu \mathrm{m}$. Error bars indicate SEM.

selection of R7 axons (Ferguson et al., 2009). Tutl is the fly homolog of Dasm1 in mice and IgSF9 in humans (Bodily et al., 2001; Doudney et al., 2002; Shi et al., 2004; Cameron and Rao, 2010). In addition to its role in axonal tiling, Tutl is also required for dendrite self- avoidance in the peripheral nervous system (Long et al., 2009). How Tutl negatively regulates adhesion between adjacent same-type neurites in tiling and selfavoidance, however, remains unknown. Since the R7 tiling phenotype caused by $\mathrm{Bdl}$ overexpression resembles that of tutl loss-of-function mutants, we speculate that Tutl downregulates R7 axon-axon adhesion by interacting with $\mathrm{Bdl}$. To test this, we examined potential physical and genetic interactions between $b d l$ and tutl.

Coimmunoprecipitation was performed to examine whether Tutl associates with Bdl. Tutl and Bdl-FLAG expression constructs were expressed in S2 cells. AntiFLAG antibody was used to precipitate Bdl and its interacting proteins from transfected cells. We found that anti-FLAG antibody could pull down Tutl from lysates prepared from S2 cells cotransfected with Tutl and Bdl-FLAG expression constructs, but could not pull down Tutl from lysates prepared from S2 cells transfected with Tutl expression construct only (Fig. 5A). This result suggests that Tutl forms a complex with Bdl in cultured cells.

To determine the in vivo relevance of the Tutl and Bdl association, we examined potential genetic interactions between tutl and $b d l$. We found that removing $b d l$ substantially suppressed the tiling phenotype in tutl null mutants (Fig. $5 E, F$ ). Reducing the dosage of $b d l$ by $50 \%$ also significantly suppressed the tutl tiling phenotype (Fig. $5 F)$. This effect appeared to be R-cellspecific, as similar suppression was observed when $b d l$ was selectively removed in homozygous tutl $\mathrm{R}$ cells by performing eye-specific mosaic analysis (Fig. $5 F$ ). Eye-specific expression of a $U A S-b d l$ transgene (i.e., GMR-bdl), but not a UAS$b d l$ transgene lacking Ig1-2 domains (i.e., GMR-bdl $\Delta \operatorname{Ig} 1-2)$ or a UAS-tutl transgene (i.e., GMR-tutl), in tutl and $b d l$ double-mutant R7 axons restored the tiling phenotype (Fig. 5F). Together, these results suggest strongly that Tutl mediates R7 axonal tiling by interacting with Bdl.

$b d l$ is negatively regulated by $L A R$ in controlling synaptic-layer selection of R7 axons

In addition to R7 tiling defects, overexpression of Bdl also caused mistargeting of many R7 axons into the M3 layer, where R8 axons normally terminate (Fig. 3 ). The frequencies of $\mathrm{R} 7$ targeting errors varied from $\sim 15 \%$ of $\mathrm{R} 7$ axons in transgenic lines with mild Bdl overexpression to $>80 \%$ of $\mathrm{R} 7$ axons in transgenic lines with strong Bdl overexpression (Fig. 3). Severe R7 targeting errors were also observed in $\mathrm{Bdl}$ overexpression flies at early developmental stages (i.e., 35 and 50\% pupal stages; data 
not shown). That overexpression of Bdl in both R7 and R8 axons caused a stronger R7 mistargeting phenotype than that of Bdl overexpression in R7 or R8 only (data not shown) is consistent with downregulation of R7-to-R8 adhesion facilitating synaptic-layer selection of R7 axons (Newsome et al., 2000; Maurel-Zaffran et al., 2001).

A similar R7 mistargeting phenotype was observed by removing the receptor tyrosine phosphatase LAR (Clandinin et al., 2001; Maurel-Zaffran et al., 2001). In addition to its role in stabilizing R7 axontarget interactions (Clandinin et al., 2001; Maurel-Zaffran et al., 2001), LAR is also suggested to negatively regulate an unknown cell adhesion molecule to decrease R7-to-R8 adhesion for R7 synaptic-layer selection (Maurel-Zaffran et al., 2001). To test whether LAR acts by negatively regulating Bdl, we examined potential genetic interaction between $L A R$ and $b d l$. Indeed, we found that reducing the dosage of $b d l$ substantially suppressed the R7 mistargeting phenotype in $L A R$ mutants (Fig. $6 B, C$ ). Conversely, overexpression of LAR also significantly suppressed the R7 mistargeting phenotype in transgenic flies with mild Bdl overexpression (Fig. 6D). In contrast, no such interaction was observed between $b d l$ and PTP69D (data not shown). These results suggest strongly that LAR controls R7 synaptic-layer selection at least in part by negatively regulating $\mathrm{Bdl}$.

LAR inhibits Bdl-mediated cell-cell adhesion independently of the tyrosine phosphatase activity and its binding to Liprin To further investigate the action of LAR, we examined the effect of LAR on Bdl-mediated cell-cell aggregation. Coexpression of LAR with Bdl inhibited Bdl-mediated cell aggregation (Fig. $7 C, G)$, but did not affect the expression level or cell-surface presence of Bdl (data not shown). No such inhibitory effect was observed when Bdl was coexpressed with control constructs (e.g., $U A S-R F P$; Fig. $7 G$ ). Similar inhibition was also observed when Bdl was coexpressed with a LAR mutant lacking the cytoplasmic sequence (i.e., LAR $\Delta$ cyto; Fig. $7 G$ ). This result indicates that inhibition of Bdl by LAR is independent of its tyrosine phosphatase activity or its binding to Liprin.

We then performed coaggregation experiments to examine whether coexpression of Bdl with LAR on one cell affects its adhesion to another cell expressing Bdl only. Cells coexpressing Bdl and LAR, or Bdl and LAR $\Delta$ cyto, were less likely to coaggregate with cells expressing Bdl only (Fig. 7F,H). That coexpression of Bdl with LAR only in half of transfected cells did not completely prevent the formation of coaggregates (Fig. $7 H$ ), however, suggests that effective inhibition of Bdl-mediated cell-cell adhesion requires the presence of LAR on both cells.

\section{Discussion}

In this study, we identify Bdl as a novel regulator of neuriteneurite interactions in Drosophila. Our results show that precise control of Bdl action is required for the establishment of R7 ax- onal connection pattern in the Drosophila visual system. During synaptic-layer selection, LAR negatively regulates Bdl to facilitate R7 target selection. At the R7 synaptic layer, Tutl interacts with Bdl to downregulate adhesion between adjacent R7 axonal terminals, which is necessary for the establishment of tiling pattern.

\section{Tutl interacts with Bdl in modulating homotypic axon-axon} interactions for $\mathrm{R} 7$ axonal tiling

Tiling and self-avoidance, two cellular mechanisms discovered in the early 1980s (Wassle et al., 1981; Kramer and Stent, 1985), are important for patterning neuronal circuitry (Cameron and Rao, 2010; Grueber and Sagasti, 2010). Previous studies have identified several cell-surface recognition molecules, such as Dscam, Tutl, Protocadherins, MEGF10, and MEGF11, that mediate homotypic neurite-neurite interactions in tiling and self-avoidance (Hughes et al., 2007; Matthews et al., 2007; Millard et al., 2007; Soba et al., 2007; Fuerst et al., 2008, 2009; Ferguson et al., 2009; Long et al., 2009; Kay et al., 2012; Lefebvre et al., 2012). These cell-surface recognition molecules may act by mediating homotypic repulsion or de-adhesion between adjacent same-type neurites. For instance, molecular and genetic analyses of fly Dscam 1 support a role for Dscam1 in mediating homotypic repulsion in dendritic selfavoidance (Hughes et al., 2007; Matthews et al., 2007; Soba et al., 2007), whereas mammalian Dscams appear to mediate de-adhesion by interfering with some unknown cell-type-specific cell adhesion molecules (Fuerst et al., 2009). The exact mechanisms by which these cell-surface recognition molecules mediate homotypic repulsion or de-adhesion, however, remains elusive. 

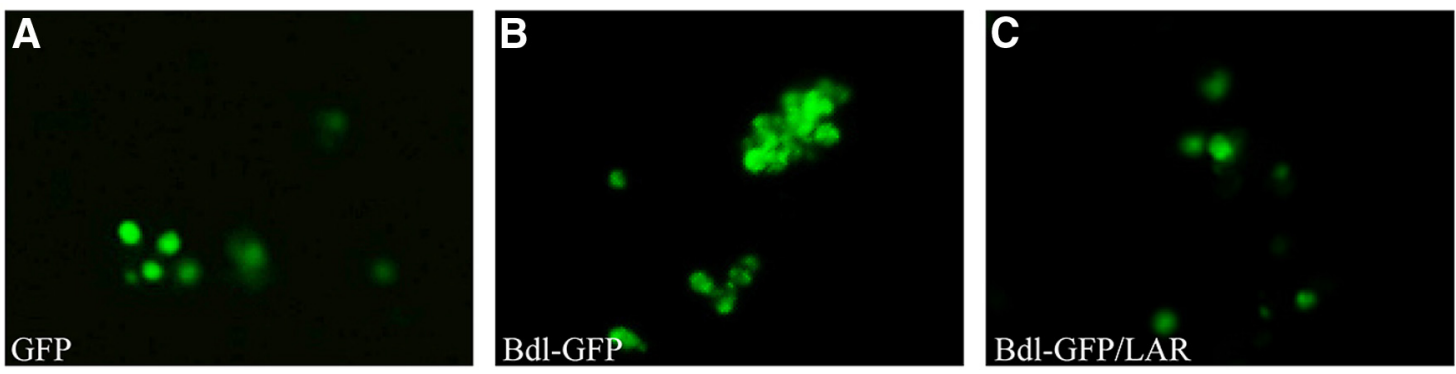

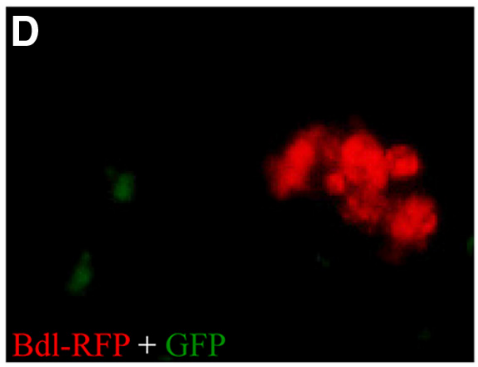

G

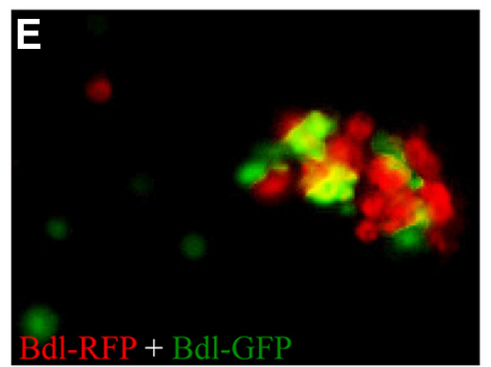

H
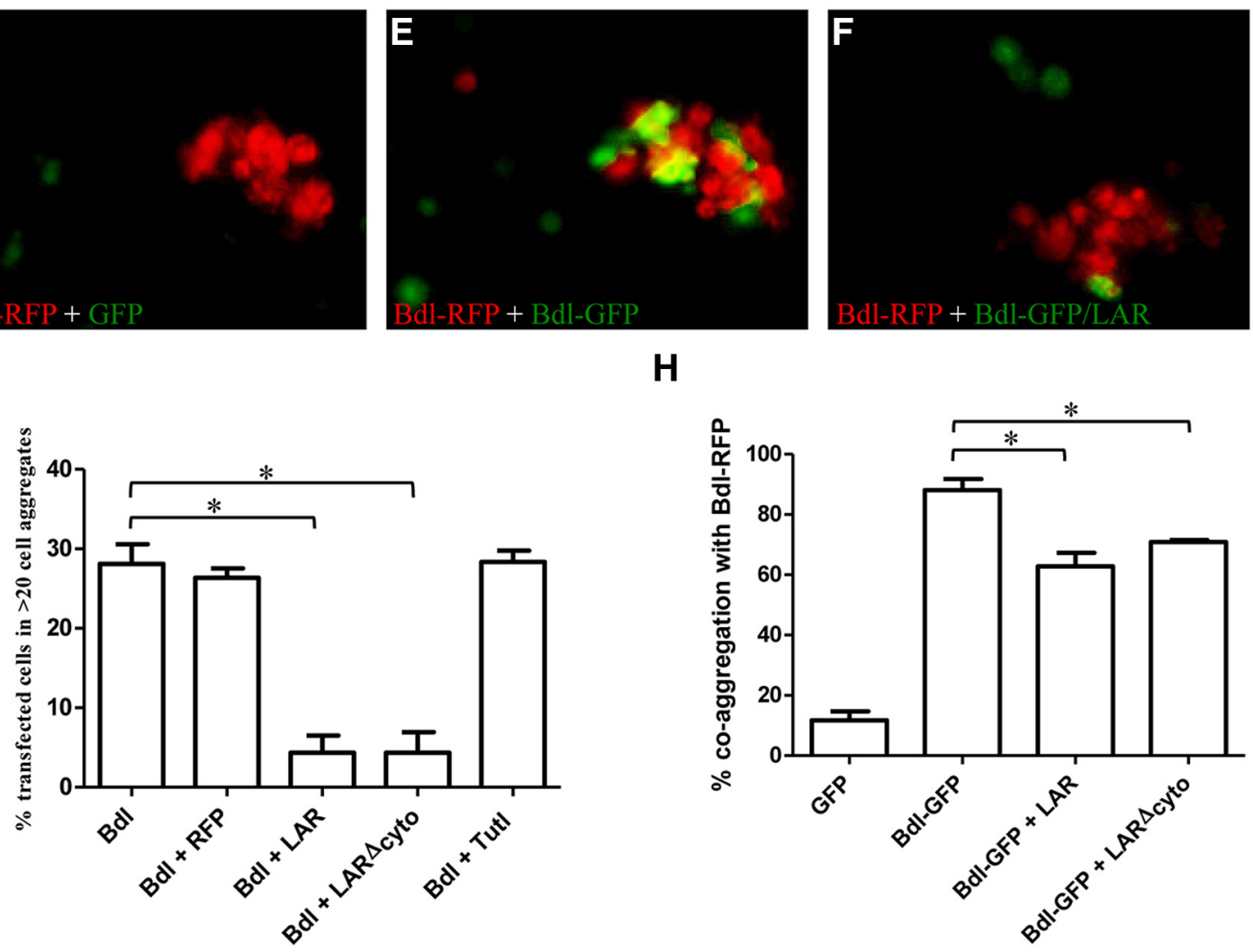

Figure 7. LAR inhibited Bdl-mediated cell-cell adhesion in cultured cells. A, S2 cells transfected with GFP vector. B, S2 cells expressing Bdl-GFP formed large cell aggregates (>20 cells). C, LAR inhibited Bdl-induced cell aggregation. LAR was tagged with the HA epitope. D, GFP-transfected cells did not coaggregate with Bdl-RFP-transfected cells. $E$, Coaggregation of Bdl-GFP- and Bdl-RFP-transfected cells. $\boldsymbol{F}$, The formation of coaggregates was reduced when LAR was coexpressed with Bdl-GFP in half of cell populations. $G$, Quantification of cell-cell aggregation data. Coexpression of UAS-Bdl-GFP with UAS-LAR ( ${ }^{*} p=0.0009$ ) or UAS-LAR- $\Delta$ cyto ( $\left.{ }^{*} p=0.0016\right)$ inhibited Bdl-mediated cell- cell aggregation, whereas no inhibition was observed when UAS-bdl-GFP was coexpressed with a UAS-RFP or a UAS-tutl construct $(p>0.05)$. Three independent experiments were performed. $\boldsymbol{H}$, Quantification of coaggregation data. The percentage of Bdl-GFP and Bdl-RFP coaggregates was decreased significantly if half of the cell population in the mixture coexpressed Bdl and LAR $\left({ }^{*} p=0.005\right)$ or Bdl and LAR- $\Delta$ cyto $\left({ }^{*} p=0.03\right)$. Three independent experiments were performed. Error bars indicate SEM.

Several lines of evidence implicate Bdl as a target of Tutl in regulating R7 axonal tiling. First, overexpression of Bdl induced an R7 tiling phenotype similar to that in tutl mutants. Second, Tutl associates with Bdl in cultured cells. And third, loss of $b d l$ rescued the tiling phenotype in tutl mutants. We propose that Tutl-mediated surface recognition counteracts the affinity between adjacent R7 axonal terminals by interacting with Bdl. The association of Tutl with Bdl may downregulate the level and/or adhesive activity of $\mathrm{Bdl}$, thus allowing the separation of adjacent R7 axonal terminals. Since co-overexpression of Tutl and Bdl did not affect Bdl-mediated cell-cell aggregation in culture (Fig. $7 G$ ) nor the Bdl-overexpression-induced tiling phenotype in flies (data not shown), we speculate that the regulation of Bdl by Tutl requires the involvement of additional regulatory molecules. Future studies are needed to determine the exact mechanism by which Tutl downregulates the function of Bdl. It will also be of interest to determine whether other cell-surface recognition mol- ecules implicated in tiling and self-avoidance (e.g., Dscam and Protocadherins), function similarly to modulate certain cell adhesion molecules.

\section{LAR negatively regulates Bdl-mediated heterotypic axon-} axon interactions in controlling synaptic-layer selection of R7 axons

The receptor tyrosine phosphatase LAR and its mammalian homologs have been shown to play important roles in axon guidance, neuronal target selection, and presynaptic development (Chagnon et al., 2004; Stryker and Johnson, 2007). In the developing Drosophila visual system, LAR is required for target selection of R1-R6 axons in the lamina, and synaptic-layer selection of R7 axons in the medulla (Clandinin et al., 2001; Maurel-Zaffran et al., 2001). The action of LAR in R7 synaptic-layer selection reportedly involves both stabilization of axon-target interactions and down-regulation of adhesion between R7 and R8 axons 
(Clandinin et al., 2001; Maurel-Zaffran et al., 2001). LARmediated axon-target interactions may involve the binding between LAR on R7 axons and an unknown ligand in the target layer, which in turn modulates the interaction between LAR and its cytoplasmic domain-binding partner Liprin to stabilize axontarget interactions (Hofmeyer et al., 2006). It is also reported that LAR negatively regulates an unknown cell adhesion molecule to decrease adhesion between R7 and R8 axons for facilitating synaptic-layer selection of R7 axons (Maurel-Zaffran et al., 2001).

Our results suggest strongly that LAR downregulates adhesion between $\mathrm{R} 7$ and $\mathrm{R} 8$ axons by negatively regulating Bdl. That LAR inhibited Bdl-mediated cell-cell adhesion without affecting the level of Bdl (Fig. 7; data not shown) suggests that LAR inhibits adhesive activity of Bdl. Although the role of LAR in mediating axon-target interactions requires its binding to Liprin via the cytoplasmic domain (Hofmeyer et al., 2006; Prakash et al., 2009), negative regulation of Bdl by LAR appears to involve a Liprinindependent mechanism. This is supported by our in vitro analysis showing that a LAR mutant lacking the cytoplasmic domain also inhibited Bdl-mediated adhesion. Consistently, a previous study showed that R8-specific expression of a truncated LAR mutant lacking the cytoplasmic domain in $L A R$ mutants could partially rescue the $\mathrm{R} 7$ mistargeting phenotype (Maurel-Zaffran et al., 2001). LAR may directly modulate Bdl to downregulate R7-R8 adhesion, or act indirectly by interacting with other proteins. Future studies are needed to distinguish between these possibilities.

\section{The role of Bdl-mediated neurite-neurite interactions in visual circuit development}

Although negative regulation of Bdl-mediated axon-axon interactions is necessary for R7 synaptic-layer selection and tiling, it remains unclear how the presence of Bdl contributes to the formation of the R-cell axonal projection pattern in the fly visual system. Cell adhesion molecules, such as NCAM/FasII and L1CAM/Neuroglian, have been shown to mediate selective fasciculation in axonal pathfinding (Thanos et al., 1984; Keilhauer et al., 1985; Tomasiewicz et al., 1993). Similarly, Bdl-mediated axonaxon interactions may facilitate the projections of R7 and/or $\mathrm{R} 1-\mathrm{R} 6$ axons along the pioneer $\mathrm{R} 8$ axon. That the $\mathrm{R}$-cell projection pattern remained normal in $b d l$ mutants may be due to the presence of redundant genes. Functional redundancy among different cell adhesion molecules seems to be common in the developing nervous system (Giagtzoglou et al., 2009), which may account for no or subtle phenotypes in mutants defective in a number of cell adhesion molecules (Tomasiewicz et al., 1993; Varoqueaux et al., 2006).

In conclusion, our present study identifies $\mathrm{Bdl}$ as a novel and important regulator of neurite-neurite interactions in the developing visual system. Tuning of Bdl-mediated axon-axon interactions in axonal tiling and synaptic-layer selection presents an excellent example for modulating the action of cell adhesion molecules in ensuring accuracy in circuit assembly. It is highly likely that similar mechanisms are employed for circuit assembly in mammalian nervous systems.

\section{References}

Bodily KD, Morrison CM, Renden RB, Broadie K (2001) A novel member of the Ig superfamily, turtle, is a CNS-specific protein required for coordinated motor control. J Neurosci 21:3113-3125. Medline

Cameron S, Rao Y (2010) Molecular mechanisms of tiling and selfavoidance in neural development. Mol Brain 3:28. CrossRef Medline

Chagnon MJ, Uetani N, Tremblay ML (2004) Functional significance of the
LAR receptor protein tyrosine phosphatase family in development and diseases. Biochem Cell Biol 82:664-675. CrossRef Medline

Clandinin TR, Zipursky SL (2000) Afferent growth cone interactions control synaptic specificity in the Drosophila visual system. Neuron 28:427436. CrossRef Medline

Clandinin TR, Lee CH, Herman T, Lee RC, Yang AY, Ovasapyan S, Zipursky SL (2001) Drosophila LAR regulates R1-R6 and R7 target specificity in the visual system. Neuron 32:237-248. CrossRef Medline

Doudney K, Murdoch JN, Braybrook C, Paternotte C, Bentley L, Copp AJ, Stanier P (2002) Cloning and characterization of Igsf9 in mouse and human: a new member of the immunoglobulin superfamily expressed in the developing nervous system. Genomics 79:663-670. CrossRef Medline

Ferguson K, Long H, Cameron S, Chang WT, Rao Y (2009) The conserved Ig superfamily member Turtle mediates axonal tiling in Drosophila. J Neurosci 29:14151-14159. CrossRef Medline

Fuerst PG, Koizumi A, Masland RH, Burgess RW (2008) Neurite arborization and mosaic spacing in the mouse retina require DSCAM. Nature 451:470-474. CrossRef Medline

Fuerst PG, Bruce F, Tian M, Wei W, Elstrott J, Feller MB, Erskine L, Singer JH, Burgess RW (2009) DSCAM and DSCAML1 function in self-avoidance in multiple cell types in the developing mouse retina. Neuron 64:484497. CrossRef Medline

Giagtzoglou N, Ly CV, Bellen HJ (2009) Cell adhesion, the backbone of the synapse: "vertebrate" and "invertebrate" perspectives. Cold Spring Harb Perspect Biol 1:a003079. CrossRef Medline

Grueber WB, Sagasti A (2010) Self-avoidance and tiling: Mechanisms of dendrite and axon spacing. Cold Spring Harb Perspect Biol 2:a001750. CrossRef Medline

Hadjieconomou D, Timofeev K, Salecker I (2011) A step-by-step guide to visual circuit assembly in Drosophila. Curr Opin Neurobiol 21:76-84. CrossRef Medline

Hofmeyer K, Maurel-Zaffran C, Sink H, Treisman JE (2006) Liprin-alpha has LAR-independent functions in R7 photoreceptor axon targeting. Proc Natl Acad Sci U S A 103:11595-11600. CrossRef Medline

Hughes ME, Bortnick R, Tsubouchi A, Bäumer P, Kondo M, Uemura T, Schmucker D (2007) Homophilic Dscam interactions control complex dendrite morphogenesis. Neuron 54:417-427. CrossRef Medline

Kay JN, Chu MW, Sanes JR (2012) MEGF10 and MEGF11 mediate homotypic interactions required for mosaic spacing of retinal neurons. Nature 483:465-469. CrossRef Medline

Keilhauer G, Faissner A, Schachner M (1985) Differential inhibition of neurone-neurone, neurone-astrocyte and astrocyte-astrocyte adhesion by L1, L2 and N-CAM antibodies. Nature 316:728-730. CrossRef Medline

Kramer AP, Stent GS (1985) Developmental arborization of sensory neurons in the leech Haementeria ghilianii. II. Experimentally induced variations in the branching pattern. J Neurosci 5:768-775. Medline

Lee CH, Herman T, Clandinin TR, Lee R, Zipursky SL (2001) N-cadherin regulates target specificity in the Drosophila visual system. Neuron 30: 437-450. CrossRef Medline

Lee RC, Clandinin TR, Lee CH, Chen PL, Meinertzhagen IA, Zipursky SL (2003) The protocadherin Flamingo is required for axon target selection in the Drosophila visual system. Nat Neurosci 6:557-563. CrossRef Medline

Lefebvre JL, Kostadinov D, Chen WV, Maniatis T, Sanes JR (2012) Protocadherins mediate dendritic self-avoidance in the mammalian nervous system. Nature 488:517-521. CrossRef Medline

Long H, Ou Y, Rao Y, van Meyel DJ (2009) Dendrite branching and selfavoidance are controlled by Turtle, a conserved IgSF protein in Drosophila. Development 136:3475-3484. CrossRef Medline

Matthews BJ, Kim ME, Flanagan JJ, Hattori D, Clemens JC, Zipursky SL, Grueber WB (2007) Dendrite self-avoidance is controlled by Dscam. Cell 129:593-604. CrossRef Medline

Maurel-Zaffran C, Suzuki T, Gahmon G, Treisman JE, Dickson BJ (2001) Cell-autonomous and -nonautonomous functions of LAR in R7 photoreceptor axon targeting. Neuron 32:225-235. CrossRef Medline

Meinertzhagen, IA, and Hanson, TE (1993) The development of the optic lobe. In: The development of Drosophila melanogaster (Bates M, Arias AM, eds), pp 1363-1492. Cold Spring Harbor, New York: Cold Spring Harbor UP.

Melnattur KV, Lee CH (2011) Visual circuit assembly in Drosophila. Dev Neurobiol 71:1286-1296. CrossRef Medline 
Millard SS, Flanagan JJ, Pappu KS, Wu W, Zipursky SL (2007) Dscam2 mediates axonal tiling in the Drosophila visual system. Nature 447:720724. CrossRef Medline

Newsome TP, Asling B, Dickson BJ (2000) Analysis of Drosophila photoreceptor axon guidance in eye-specific mosaics. Development 127:851-860. Medline

Parks AL, Cook KR, Belvin M, Dompe NA, Fawcett R, Huppert K, Tan LR, Winter CG, Bogart KP, Deal JE, Deal-Herr ME, Grant D, Marcinko M, Miyazaki WY, Robertson S, Shaw KJ, Tabios M, Vysotskaia V, Zhao L, Andrade RS, et al. (2004) Systematic generation of high-resolution deletion coverage of the Drosophila melanogaster genome. Nat Genet 36: 288-292. CrossRef Medline

Prakash S, Caldwell JC, Eberl DF, Clandinin TR (2005) Drosophila $\mathrm{N}$-cadherin mediates an attractive interaction between photoreceptor axons and their targets. Nat Neurosci 8:443-450. Medline

Prakash S, McLendon HM, Dubreuil CI, Ghose A, Hwa J, Dennehy KA, Tomalty KM, Clark KL, Van Vactor D, Clandinin TR (2009) Complex interactions amongst N-cadherin, DLAR, and Liprin-alpha regulate Drosophila photoreceptor axon targeting. Dev Biol 336:10-19. CrossRef Medline

Sanes JR, Zipursky SL (2010) Design principles of insect and vertebrate visual systems. Neuron 66:15-36. CrossRef Medline

Shi SH, Cheng T, Jan LY, Jan YN (2004) The immunoglobulin family member dendrite arborization and synapse maturation 1 (Dasm1) controls excitatory synapse maturation. Proc Natl Acad Sci U S A 101:1334613351. CrossRef Medline
Shinza-Kameda M, Takasu E, Sakurai K, Hayashi S, Nose A (2006) Regulation of layer-specific targeting by reciprocal expression of a cell adhesion molecule, capricious. Neuron 49:205-213. CrossRef Medline

Soba P, Zhu S, Emoto K, Younger S, Yang SJ, Yu HH, Lee T, Jan LY, Jan YN (2007) Drosophila sensory neurons require Dscam for dendritic selfavoidance and proper dendritic field organization. Neuron 54:403-416. CrossRef Medline

Stryker E, Johnson KG (2007) LAR, liprin alpha and the regulation of active zone morphogenesis. J Cell Sci 120:3723-3728. CrossRef Medline

Thanos S, Bonhoeffer F, Rutishauser U (1984) Fiber-fiber interaction and tectal cues influence the development of the chicken retinotectal projection. Proc Natl Acad Sci U S A 81:1906-1910. CrossRef Medline

Timofeev K, Joly W, Hadjieconomou D, Salecker I (2012) Localized netrins act as positional cues to control layer-specific targeting of photoreceptor axons in Drosophila. Neuron 75:80-93. CrossRef Medline

Tomasiewicz H, Ono K, Yee D, Thompson C, Goridis C, Rutishauser U, Magnuson T (1993) Genetic deletion of a neural cell adhesion molecule variant (N-CAM-180) produces distinct defects in the central nervous system. Neuron 11:1163-1174. CrossRef Medline

Varoqueaux F, Aramuni G, Rawson RL, Mohrmann R, Missler M, Gottmann K, Zhang W, Südhof TC, Brose N (2006) Neuroligins determine synapse maturation and function. Neuron 51:741-754. CrossRef Medline

Wässle H, Peichl L, Boycott BB (1981) Dendritic territories of cat retinal ganglion cells. Nature 292:344-345. CrossRef Medline 